WATANABE, Y. AND K. TAKAMATSU

KODAI MATH. SEM. REP

25 (1973), 297-306

\title{
ON A $K$-SPACE OF CONSTANT HOLOMORPHIC SECTIONAL CURVATURE
}

\author{
By Yoshiyuki Watanabe and Kichiro Takamatsu
}

\section{Introduction.}

It is well known that a Kähler space of constant holomorphic sectional curvature is an Einstein space (Yano [13]). The main purpose of the present paper is to generalize this result to a $K$-space (Theorem 4.4). Preliminary facts will be given in $\S 2$. In $\S 3$, we shall prepare some lemmas for the proof of main theorem. Particularly, we shall prove some lemmas about the $K$-space of constant holomorphic sectional curvature. In $\S 4$, we shall prove the main theorem and some related theorems on the generalized Chern-form.

\section{Preliminaries.}

Let $M$ be an $n$-dimensional $(n>2)$ almost Hermitian manifold with Hermitian structure $\left(F_{j}{ }^{2}, g_{j i}\right)$, i.e., with an almost complex structure $F_{j}{ }^{i}$ and a positive definite Riemannian metric tensor $g_{j i}$ satisfying

$$
\begin{aligned}
F_{j}{ }^{a} F_{a}{ }^{i} & =-\delta_{j}^{i}, \\
g_{t s} F_{j}{ }^{t} F_{\imath}{ }^{s} & =g_{j i} .
\end{aligned}
$$

If an almost Hermitian structure satisfies

$$
\nabla_{k} F_{j i}+\nabla_{j} F_{k i}=0,
$$

where $\nabla_{j}$ denotes the operator of covariant differentiation with respect to the Riemannian connection and $F_{j i}=F_{j}{ }^{t} g_{t i}$, then the manifold is called a $K$-space (or Tachibana space, or nearly Kähler manifold).

Now, in a $K$-space let $R_{k j i}{ }^{h}, R_{j i}=R_{h j i}{ }^{h}$ and $R=g^{j i} R_{j i}$ be Riemannian curvature tensor, Ricci tensor and scalar curvature respectively. Then we have the following identities [7], [8]:

$$
\begin{gathered}
\nabla_{j} F_{i h}+F_{j}{ }^{b} F_{i}{ }^{a} \nabla_{b} F_{a h}=0, \\
F_{h k} \nabla^{t} \nabla_{t} F_{j}{ }^{h}=R_{k j}-R^{*}{ }_{j k}, \quad \text { or } \quad \nabla^{t} \nabla_{t} F_{j}{ }^{h}=F^{h l}\left(R_{j l}-R^{*}{ }_{l j}\right),
\end{gathered}
$$

Received September 13, 1972. 
where $\nabla^{t}=g^{t a} \nabla_{a}$ and $R^{*}{ }_{j i}=(1 / 2) F^{b a} R_{b a t i} F_{j}{ }^{t}$,

$$
R_{j i}=F_{\jmath}{ }^{b} F_{\imath}{ }^{a} R_{b a}, \quad R^{*}{ }_{j i}=F_{\jmath}{ }^{b} F_{\imath}{ }^{a} R^{*}{ }_{b a}
$$

$$
\begin{gathered}
R^{*}{ }_{j i}=R_{\imath \jmath}, \\
\nabla_{j} F_{t s}\left(\nabla_{i} F^{t s}\right)=R_{j i}-R^{*}{ }_{j i},
\end{gathered}
$$

where $F^{j i}=F_{t}{ }^{2} g^{t j} ;$ and

$$
\nabla_{j} F_{i h}\left(\nabla^{j} F^{i h}\right)=R-R^{*}=\text { constant }>0,
$$

where $R^{*}=g^{j i} R^{*}{ }_{j i}$.

Since we have $T^{k j i} \nabla_{k} R_{j i h l}=0$ for any skew-symmetric tensor $T^{k j i}$, we get

$$
\left(\nabla^{k} F^{j i}\right) \nabla_{k} R_{j i h l}=0 .
$$

As $(1 / 2) \nabla_{i} R^{*}=\nabla^{j} R^{*}{ }_{j i}$ in a $K$-space [5], we have

$$
\nabla^{k}\left(R_{i k}-R^{*}{ }_{i k}\right)=\frac{1}{2} \nabla_{i}\left(R-R^{*}\right)=0 .
$$

\section{Some lemmas.}

In general, it is well known that the differential form

$$
\hat{K}=\hat{K}_{j i} d x^{\jmath} \wedge d x^{\imath}
$$

is closed in any almost Hermitian manifold, where

$$
\hat{K}_{j i}=2 R_{j i r}{ }^{t} F_{t}^{r}-F_{s}{ }^{t}\left(\nabla_{j} F_{r}^{s}\right) \nabla_{i} F_{t}^{r},
$$

which is called the generalized Chern-form [8].

In a $K$-space, taking account of (2.8), we have

$$
\hat{K}_{j i}=F_{j}^{r}\left(5 R^{*}{ }_{r i}-R_{r i}\right) .
$$

Recently, A. Gray proved the following

Lemma 3. 1. (Gray [1]) In a $K$-space, the relation

$$
\begin{aligned}
& R_{j i s t}-R_{b a s t} F_{j}^{b} F_{\imath}^{a}=-\left(\nabla_{j} F_{\imath}^{r}\right) \nabla_{s} F_{t r}, \\
& R_{j i h k}-F_{j}^{a} F_{i}^{b} F_{h}^{c} F_{k}^{d} R_{a b c d}=0
\end{aligned}
$$

\section{hold good.}

Moreover, the present authors proved the following

Lemma 3. 2. (Takamatsu and Watanabe [11]) In a K-space, we have 


$$
\nabla_{h} S_{s i}=-\frac{1}{2}\left\{S_{r i}\left(\nabla^{r} F_{s m}\right) F_{h}{ }^{m}+S_{r s}\left(\nabla_{i} F_{m}^{r}\right) F_{h}{ }^{m}\right\}
$$

where $S_{j i}=R_{j i}-R^{*}{ }_{j i}$.

Now we have the following

LEMMA 3. 3. In a $K$-space, the relation

$$
S^{j i}\left(R_{k j i h}-5 R_{k j b a} F_{i}^{b} F_{h}^{a}\right)=0
$$

holds good.

Proof. If we transvect (3.2) with $\nabla_{h} F^{j i}$, then we have, taking account of (2. 4) and (2.8),

$$
2\left(\nabla_{h} F^{j i}\right) R_{j i s t}=-S_{h}^{r} \nabla_{s} F_{t r} .
$$

Applying $\nabla^{h}$ to this equation, we have, by (2.10) and (2.11),

$$
2\left(\nabla^{h} \nabla_{h} F^{j i}\right) R_{j i s t}=-S_{h}{ }^{r} \nabla^{h} \nabla_{s} F_{t r},
$$

or by Ricci's identity and $S^{h r} \nabla_{s} \nabla_{h} F_{t r}=0$,

$$
\begin{aligned}
2\left(\nabla^{h} \nabla_{h} F^{j i}\right) R_{j i s t} & =S_{h}{ }^{r}\left(-\nabla_{s} \nabla^{h} F_{t r}+R^{h}{ }_{s t}^{a} F_{a r}+R^{h}{ }_{s r}^{a} F_{t a}\right) \\
& =S_{h}{ }^{r}\left(R^{h}{ }_{s a t} F_{r}{ }^{a}-R^{h}{ }_{s a r} F_{t}^{a}\right) .
\end{aligned}
$$

Transvecting (3.6) with $F_{k}^{t}$, we have,

$$
2 F_{k}^{t}\left(\nabla^{h} \nabla_{h} F^{j i}\right) R_{j i s t}=S^{h r}\left(R_{h s k r}-R_{h s t a} F_{k}^{t} F_{r}^{a}\right) .
$$

Substituting (2.5) into (3.7), we obtain

$$
2 F_{k}{ }^{t} F_{a}^{j} S^{a i} R_{j i s t}=S^{h r}\left(R_{h s k r}-R_{h s t a} F_{k}^{t} F_{r}{ }^{a}\right),
$$

or by Bianchi's identity,

$$
2 F_{k}{ }^{t} F_{a}{ }^{j} S^{a i}\left(R_{i t j s}+R_{t j i s}\right)=S^{h r}\left(R_{h s k r}-R_{h s t a} F_{k}{ }^{t} F_{r}{ }^{a}\right) .
$$

Moreover, making use of $F_{a}^{j} S^{a_{\imath}}=-F_{a}^{i} S^{a_{\jmath}}$, (3.8) reduces to

$$
4 F_{k}^{t} F_{a}^{j} R_{t j i s} S^{a i}=S^{h r}\left(R_{h s k r}-R_{h s t a} F_{k}{ }^{t} F_{r}^{a}\right)
$$

from which we have (3.5).

Transvecting (3.5) with $g^{k h}$, we have the following

Lemma 3. 4. (Takamatsu [10]) In a $K$-space, the relation

$$
S^{j i}\left(R_{j i}-5 R^{*}{ }_{j i}\right)=0
$$

\section{holds good.}


Lemma 3.5. In a K-space, we have

$$
\left(R_{k j i h}-R_{k j b a} F_{i}^{b} F_{h}^{a}\right) S^{j i}=\frac{1}{4}\left(3 R_{k r}+R_{k r}{ }_{k}\right) S_{h}{ }^{r} .
$$

Proof. By Ricci's identity, we have

$$
\nabla_{h} \nabla_{i} S_{s l}-\nabla_{i} \nabla_{h} S_{s l}=R_{h i m s} S_{l}{ }^{m}+R_{h \imath m l} S_{s}{ }^{m} .
$$

Transvecting (3.12) with $g^{i l}$, we have, by virture of (2.11),

$$
R_{h \imath m s} S^{\imath m}=R_{h m} S_{s}{ }^{m}-\nabla^{i} \nabla_{h} S_{s i} \text {. }
$$

Now, applying $\nabla^{i}$ to (3. 4), we have

$$
-2 \nabla^{i} \nabla_{h} S_{s i}=S_{r i}\left(\nabla^{i} \nabla^{r} F_{s m}\right) F_{h}{ }^{m}+S_{r i}\left(\nabla^{r} F_{s m}\right) \nabla^{i} F_{h}{ }^{m}
$$

$$
+\nabla^{i} S_{s r}\left(\nabla_{i} F_{m}{ }^{r}\right) F_{h}{ }^{m}+S_{r s}\left(\nabla^{i} \nabla_{i} F_{m}{ }^{r}\right) F_{h}{ }^{m}+S_{s r}\left(\nabla_{i} F_{m}{ }^{r}\right) \nabla^{i} F_{h}{ }^{m} .
$$

Let us calculate the right hand side of (3.14). By Ricci's identity and (3.2), we have

$$
\begin{aligned}
\nabla_{i} \nabla_{s} F_{m}{ }^{r}-\nabla_{s} \nabla_{i} F_{m}{ }^{r} & =R_{\imath s a}{ }^{r} F_{m}{ }^{a}-R_{i s m}{ }^{b} F_{b}^{r} \\
& =F_{m}{ }^{a}\left(R_{i s a}{ }^{r}-R_{i s t b} F^{r b} F_{a}{ }^{t}\right) \\
& =F_{m}{ }^{a}\left(-\nabla_{i} F_{s t}\right) \nabla_{a} F^{r t} .
\end{aligned}
$$

Multiplying both sides of (3.15) by $S_{r}^{i} F_{h}{ }^{m}$, we have

$$
S^{i}{ }_{r} F_{h}{ }^{m} \nabla_{i} \nabla_{s} F_{m}{ }^{r}=S^{i}{ }_{r}\left(\nabla_{i} F_{s t}\right) \nabla_{h} F^{r t} .
$$

Thus, the first term of the right hand side of (3.14) reduces to

$$
S_{r i}\left(\nabla^{i} \nabla^{r} F_{s m}\right) F_{h}{ }^{m}=-\nabla^{i} F_{s}{ }^{t}\left(\nabla^{r} F_{h t}\right) S_{r i} \text {. }
$$

For the third term, by (3.2), (3.4), (3.9) and $\left(\nabla^{i} F_{m}{ }^{r}\right) F_{h}{ }^{m}=\left(\nabla^{r} F_{h}{ }^{m}\right) F_{m}{ }^{2}$, we have

$$
\begin{aligned}
\nabla_{\imath} S_{r s}\left(\nabla^{i} F_{m}^{r}\right) F_{h}{ }^{m} & =-\frac{1}{2}\left\{S_{t s}\left(\nabla^{t} F_{r l}\right) F_{i}^{l}+S_{t r}\left(\nabla_{s} F_{l}^{t}\right) F_{i}{ }^{l}\right\}\left(\nabla^{i} F_{m}^{r}\right) F_{h}{ }^{m} \\
& =-\frac{1}{2}\left\{S_{t s}\left(\nabla_{r} F_{m}^{t}\right)-S_{t r}\left(\nabla_{s} F_{m}{ }^{t}\right)\right\} \nabla^{r} F_{h}{ }^{m} \\
& =-\frac{1}{2}\left\{S_{t s} S_{h}^{t}+S^{t r}\left(\nabla_{t} F_{s m}\right) \nabla_{h} F_{r}{ }^{m}\right\} \\
& =-\frac{1}{2}\left\{S_{t s} S_{h}{ }^{t}-S^{t r}\left(R_{t s h r}-R_{t s b a} F_{r}^{a} F_{h}^{b}\right)\right\} \\
& =-\frac{1}{2} S_{t s} S_{h}{ }^{t}+2 F_{h}{ }^{t} F_{a}^{j} R_{t j b s} S^{a b}
\end{aligned}
$$


The fourth term, making use of (2.5), reduces to

$$
S_{r s}\left(\nabla^{i} \nabla_{i} F_{m}{ }^{r}\right) F_{h}{ }^{m}=S_{r s} S_{h}{ }^{r} .
$$

Substituting (3.16), (3.17) and (3.18) into (3.14), we have

$$
\nabla^{i} \nabla_{h} S_{s \imath}=\frac{1}{4} S_{s}^{r} S_{h r}-F_{h}{ }^{t} F_{a}^{j} R_{t j i s} S^{a \imath} .
$$

Thus, making use of (3.19), from (3.13), we have

$$
R_{h m \imath s} S^{i m}=R_{h m} S_{s}{ }^{m}-\frac{1}{4} S_{h m} S_{s}{ }^{m}+F_{h}{ }^{t} F_{m}{ }^{j} R_{t j i s} S^{m \imath},
$$

or

$$
\left(R_{h m l s}-F_{h}{ }^{t} F_{m}{ }^{j} R_{t j i s}\right) S^{i m}=\left(R_{h m}-\frac{1}{4} S_{h m}\right) S_{s}{ }^{m},
$$

from which we have (3. 11).

Lemma 3. 6. In a $K$-space, we have

$$
\begin{aligned}
S^{j i} R_{k j i h} & =\frac{5}{16} T_{k h}, \\
S^{j i} R_{k j b a} F_{i}^{b} F_{h}{ }^{a} & =\frac{1}{16} T_{k l}, \\
S^{h j} F_{h}{ }^{r} F_{k}^{q} R_{r j i q} & =\frac{1}{8} T_{i k},
\end{aligned}
$$

where $T_{k h}=T_{h k}=\left(3 R_{k r}+R_{k r}^{*}\right) S_{h}{ }^{r}$.

Proof. First, by (3.11) and $S_{j i}=S_{i j}, T_{k h}=T_{h k}$ is, easily verified. (3.20) and (3.21) immediately follow from simultaneous equation (3.5) and (3.11) with respect to $S^{j i} R_{k j i h}, S^{j i} R_{k j b a} F_{\imath}^{b} F_{h}{ }^{a}$. For (3.22), making use of Bianchi's identity, (2.6) and (3. 21), we have

$$
\begin{aligned}
S^{h j} F_{h}^{r} F_{k}^{q} R_{r j i q} & =S^{h j} F_{h}^{r} F_{k}^{q}\left(-R_{j i r q}-R_{\imath r \jmath q}\right) \\
& =S^{h j} F_{h}^{r} F_{k}^{q} R_{\imath \jmath r q}-S^{h j} F_{h}^{r} F_{k}^{q} R_{q \jmath r \imath} \\
& =S^{h j} F_{h}^{r} F_{k}^{q} R_{\imath j r q}+S^{h r} F_{h}^{j} F_{k}^{q} R_{q \jmath r \imath} \\
& =\frac{1}{16} T_{i k}+\frac{1}{16} T_{i k}=\frac{1}{8} T_{i k} .
\end{aligned}
$$

LemMA 3.7. In a K-space, we have

$$
\nabla_{k} S_{j i}\left(\nabla^{k} S^{j i}\right)=\frac{1}{8}\left(R_{b h}-5 R^{*}{ }_{b h}\right) S^{b i} S^{h}{ }^{\prime} .
$$


Proof. Calculating the square of both sides of (3.4), we have

$$
2 V_{k} S_{j i}\left(\nabla^{k} S^{j i}\right)=S^{h} S^{b \imath}\left(\nabla_{h} F_{\jmath m}\right) \nabla_{b} F^{j m}+S^{h i} S^{b j}\left(\nabla_{h} F_{\jmath m}\right) V_{b} F_{\imath}{ }^{m} .
$$

Substituting (2.8) and (3.2) into (3.25), by (3.20) and (3.21), we obtain

$$
\begin{aligned}
2 \nabla_{k} S_{j i}\left(\nabla^{k} S^{j i}\right) & =S^{h}{ }^{h} S^{b i} S_{h b}-S^{h i} S^{b j}\left(R_{h j b i}-R_{h \jmath p q} F_{b}{ }^{p} F_{i}^{q}\right) \\
& =S^{h}{ }_{\imath} S^{b i} S_{h b}-S^{h i}\left(\frac{5}{16} T_{h i}-\frac{1}{16} T_{h \imath}\right) \\
& =S^{h}{ }_{\imath}{ }^{b i} S_{h b}-\frac{1}{4} T_{h \imath} S^{h \imath} \\
& =\frac{1}{4}\left(4 R_{b h}-4 R^{*}{ }_{b h}-3 R_{h b}-R^{*}{ }_{h b}\right) S^{b i} S_{i}{ }^{h} \\
& =\frac{1}{4}\left(R_{b h}-5 R^{*}{ }_{b h}\right) S^{b \imath} S^{h}{ }_{\imath} .
\end{aligned}
$$

Lastly, we shall state some lemmas for a $K$-space of constant holomorphic sectional curvature.

Let $k$ be a holomorphic sectional curvature of an almost Hermitian space with respect to a vector $X^{h}$, that is,

$$
k=-\frac{R_{m j r h} F_{q}^{m} X^{q} F_{p}^{r} X^{p} X^{j} X^{h}}{g_{k \jmath} X^{k} X^{\jmath} g_{i h} X^{i} X^{h}}
$$

If $k=$ constant with respect to any vector at any point of the space, then the space is called a space of constant holomorphic sectional curvature. We know the following

Lemma 3. 8. (Mizusawa and Kotō [4]) If an almost Hermitian space is a space of constant holomorphic sectional curvature, then the curvature tensor of the space satisfies the following relation:

$$
\begin{aligned}
& R_{k j i h}+R_{\imath j k h}-F_{k}^{q} F_{h}^{l}\left(R_{l j i q}+R_{\imath j l q}\right) \\
& -F_{i}^{p} F_{h}^{m}\left(R_{k \jmath m p}+R_{m j k p}\right)-F_{\jmath}^{m} F_{i}^{p}\left(R_{m h k p}+R_{k h m p}\right) \\
& -F_{k}^{q} F_{j}^{l}\left(R_{i n l q}+R_{l h \imath q}\right)+F_{k}^{q} F_{i}^{p} F_{\jmath}{ }^{m} F_{h}^{l}\left(R_{m q l p}+R_{l q m p}\right) \\
= & -4 k F_{k}^{q} F_{i}^{p}\left(g_{q p} g_{h j}+g_{q h} g_{\jmath p}+g_{q j} g_{p h}\right) .
\end{aligned}
$$

For a $K$-space of constant holomorphic sectional curvature, we know the following

Lemma 3.9. (Kotō [2], Takamatsu [9]) In a K-space of constant holomorphic sectional curvature, we have

$$
R_{k r}+3 R_{k r}=(n+2) k g_{k r}
$$


or

$$
R_{k r}+3 R^{*}{ }_{k r}=\frac{R+3 R^{*}}{n} g_{k r}
$$

where $k$ is positive constant.

Leмma 3. 10. In a K-space of constant holomorphic sectional curvature $k$, we have

$$
\left(R_{k r}-5 R^{*}{ }_{k r}\right) S_{i}{ }^{r} S^{k \imath}=2 k\left(S^{2}-n S_{k \imath} S^{k i}\right)
$$

where $S=R-R^{*}$.

Proof. Transvecting (3.27) with $S^{h y}$ and making use of (3.3), we have,

$$
\begin{aligned}
& \left(R_{k j i h}+R_{\imath j k h}\right) S^{h_{\jmath}}-F_{k}^{q} F_{h}^{l}\left(R_{l j i q}+R_{\imath j l q}\right) S^{h_{\jmath}} \\
& -F_{i}{ }^{p} F_{h}{ }^{m}\left(R_{k \jmath m p}+R_{m j k p}\right) S^{h_{\jmath}}-F_{\jmath}{ }^{m} F_{i}^{p}\left(R_{m h k p}+R_{k h m p}\right) S^{h \jmath} \\
& -F_{k}^{q} F_{j}^{l}\left(R_{i h l q}+R_{l h \imath q}\right) S^{h \jmath}+\left(R_{j k h i}+R_{h k j i}\right) S^{h \jmath} \\
= & -4 k\left(g_{k i} g_{h j}+F_{k h} F_{i j}+F_{k j} F_{i h}\right) S^{h \jmath} .
\end{aligned}
$$

Then, substituting (3.20), (3.21) and (3.22) into (3.31) and making use of (2.6), we have

$$
\begin{aligned}
& -\left(\frac{5}{16} T_{k i}+\frac{5}{16} T_{i k}\right)-\left(\frac{1}{8} T_{i k}+\frac{1}{16} T_{i k}\right) \\
& -\left(\frac{1}{16} T_{k i}+\frac{1}{8} T_{k \imath}\right)-\left(\frac{1}{8} T_{k i}+\frac{1}{16} T_{k \imath}\right)-\left(\frac{1}{16} T_{i k}+\frac{1}{8} T_{i k}\right) \\
& -\frac{10}{16} T_{k \imath}=-4 k\left(S g_{k i}+2 S_{k \imath}\right) .
\end{aligned}
$$

From (3. 32), by $T_{k \imath}=T_{i k}$, we have

$$
-2 T_{k \imath}=-4 k\left(S g_{k i}+2 S_{k \imath}\right)
$$

i.e.

$$
\left(3 R_{k r}+R_{k r}^{*}\right) S_{i}^{r}=2 k\left(S g_{k i}+2 S_{k \imath}\right)
$$

Then forming (3. 33) $-2 S_{i}{ }^{r} \times(3.28)$, we have

$$
\left(R_{k r}-5 R^{*}{ }_{k r}\right) S_{i}^{r}=2 k\left(S g_{k i}-n S_{k \imath}\right) .
$$

Thus, transvecting this last equation with $S^{k \imath}$, we have

$$
\left(R_{k r}-5 R_{k r}^{*}\right) S_{i}^{r} S^{k \imath}=2 k\left(S^{2}-n S_{k \imath} S^{k i}\right) .
$$


Moreover, we know the following

Lemma 3. 11. (Sawaki and Yamagata [6]) In a K-space of constant holomorphic sectional curvature, we have

$$
\begin{gathered}
S_{j i} S^{j i}=\frac{\left(R+3 R^{*}\right)\left(R-R^{*}\right)}{2 n}, \\
\left(R_{j i}-5 R^{*}{ }_{j i}\right)\left(R^{j i}-5 R^{* j i}\right)=\frac{1}{n}\left(R+3 R^{*}\right)\left(5 R^{*}-R\right),
\end{gathered}
$$

and

$$
R^{*}<R \leqq 5 R^{*} \text {. }
$$

LEMMA 3. 12. Let $M$ be a $K$-space of constant holomorphic sectional curvature. Then, in order that $M$ is an Einstein space, it is necessary and sufficient that $R=5 R^{*}$.

Proof. For sufficiency, taking account of (3.29) and (3.35), we can easily see that $M$ is an Einstein space.

Conversely, if $M$ is an Einstein space, then from (3. 29), we have $R^{*}{ }_{j i}=\left(R^{*} / n\right) g_{j i}$. Therefore we have $R_{j i}-R^{*}{ }_{j i}=\left(\left(R-R^{*}\right) / n\right) g_{j i}$, from which we get $R=5 R^{*}$, by virture of (3. 10).

\section{Theorems.}

Theorem 4.1. Let $M$ be a K-space such that

$$
S_{j i}=R_{j i}-R^{*}{ }_{j i}=a g_{j i} .
$$

Then $M$ is an Einstein space.

Proof. Substituting (4.1) into (3. 5), we have

$$
R_{j i}=5 R^{*}{ }_{j i} .
$$

Transvecting (4.1) and (4.2) with $g^{j i}$, we have

$$
R-R^{*}=n a, \quad R=5 R^{*},
$$

respectively.

Substituting (4.2) and (4.3) into (4.1), we have

$$
R_{j i}=\frac{R}{n} g_{j i}
$$

Since a 6 -dimensional $K$-space satisfies the condition (4.1) [10], we have 
Corollary 4. 2. (Matsumoto [3]) A 6-dimensional K-space is an Einstein space.

If the generalized Chern-form $\hat{K}$ vanishes, then from (3.1), we have

$$
R_{j i}=5 R^{*}{ }_{j i} .
$$

Therefore from (3.24), we get $\nabla_{k} S_{j i}=0$. But, we know that if a symmetric tensor $E_{j i}$ is parallel in an irreducible Riemannian space, then $E_{j i}=c g_{j i}$ where $c$ is constant.

Consequently, by virture of Theorem 4 . 1 , we have

Corollary 4. 3. An irreducible K-space with vanishing generalized Chern-form $\hat{K}$ is an Einstein space.

THEOREM 4.4. A K-space of constant holomorphic sectional curvature is an Einstein space.

Proof. Substituting (3.34) into (3. 30), we have

$$
\begin{aligned}
\left(R_{k r}-5 R^{*}{ }_{k r}\right) S^{r} S^{k \imath} & =2 k\left(S^{2}-n S_{k \imath} S^{k i}\right) \\
& =2 k\left\{\left(R-R^{*}\right)^{2}-\frac{n\left(R+3 R^{*}\right)\left(R-R^{*}\right)}{2 n}\right\} \\
& =k\left(R-R^{*}\right)\left(R-5 R^{*}\right) .
\end{aligned}
$$

On the other hand, making use of (4.4), from (3.24), we have

$$
\left(\nabla_{k} S_{j i}\right) \nabla^{k} S^{j i}=\frac{1}{8} k\left(R-R^{*}\right)\left(R-5 R^{*}\right) .
$$

In the above equations (4.5), since $k>0$ and $R-R^{*}>0$, we have

$$
R-5 R^{*} \geqq 0 \text {. }
$$

Comparing (4.6) with (3.36), we have $R=5 R^{*}$.

Consequently, by virture of Lemma 3.12 , the proof of the theorem is complete.

COROLlaRy 4.5. The generalized Chern-form $\hat{K}$ of a K-space of constant holomorphic sectional curvature vanishes.

Proof. By Theorem 4.4 and Lemma 3.12, we have $R=5 R^{*}$. Hence, from (3. 35), we have $R_{j i}=5 R^{*}{ }_{j i}$ which shows that $\hat{K}=0$.

\section{REFERENCES}

[1] GRAy, A., Nearly Kähler manifolds. J. Differential Geometry 4 (1970), 465-504.

[2] Kotō, S., Curvatures in Hermitian spaces. Mem. Fac. of Educ. Niigata Unıv. 2 (1960), 151-156.

[3] Matsumoto, M., On 6-dimensional almost Tachibana spaces. Tensor N.S. 23 (1972), 250-252. 
[4] Mizusawa, H., And S. Koтō, Holomorphically projective curvature tensors in certain almost Kählerian spaces. J. Fac. Sci. Niigata Univ. 2 (1960), 33-43.

[5] Sawaki, S., On Matsushima's theorem in a compact Einstein $K$-space. Tôhoku Math. J. 13 (1961), 455-465.

[6] Sawaki, S., and M. Yamagata, Notes on almost Hermitian manifolds. To appear in Tensor N.S.

[7] Tachibana, S., On almost-analytic vectors in certain almost Hermitian manifolds. Tôhoku Math. J. 11 (1959), 351-363.

[8] - On infinitesimal conformal and projective transformations of compact $K$-spaces. Tôhoku Math. J. 13 (1961), 386-392.

[9] Takamatsu, K., Some properties of $K$-spaces with constant scalar curvature. Bull. Fac. of Educ. Kanazawa Unıv. 17 (1968), 25-27.

[10] - Some properties of 6-dimensional $K$-spaces. Kōdaı Math. Sem. Rep. 23 (1971), 215-232.

[11] Takamatsu, K., and Y. Watanabe, On conformally flat $K$-spaces. Differential Geometry in honor of K. Yano, Kinokuniya Tokyo (1972), 483-488.

[12] _ Classification of a conformally flat $K$-space. Tôhoku Math. J. 24 (1972), 435-440.

[13] Yano, K., Differential geometry on complex and almost complex spaces. Pergamon Press (1965).

TOYAMA UNIVERSITY, AND

KanAZAwa UNIVERSITY. 